\title{
A Study of Technical Efficiency of Banks in India Using Dea
}

\author{
Nandkumar $^{1}$, Archana Singh ${ }^{2}$ \\ ${ }^{1}$ Assistant Professor, Department of Humanities. DTU, \\ ${ }^{2}$ Assistant Professor, Delhi School of Management. DTU,
}

\begin{abstract}
This study uses DEA approach to estimate the technical efficiency of commercial banks in India over the years 2006-2010. The results indicate that deregulation of banking sector has led to an increase in the efficiency of commercial banks in India. Banks like Allahabad bank, Canara bank, Kotak Mahindra bank, ICICI bank and Yes bank are very efficient and show consistency in their performance. On the other hand, the performance of banks like SBI, PNB and HDFC can be a matter of concern as their efficiency scores are below satisfactory level. The major factor resulting in the poor performance of these two banks is their huge amount of deposits and operating expenses. Also, the excess number of employees is increasing their problems. So, here either these banks possess blocked/non-performing assets or are not able to make a set off between the deposits and advances. Being major banks in the country, they deserve better attention by the regulators and the administrators. The study also shows that performance of private sector banks has been better than public sector banks during the period.
\end{abstract}

Keywords: DEA; Efficiency; DMU; CCR model; Fractional LPP;

\section{Introduction}

The Banking sector plays an important role in the mobilization and allocation of savings in an economy. It plays the role of mediator between the net savers and net borrowers. The gain to the real sector depend on how efficiently the financial sector performs their function of inter -mediation. An efficient banking system contributes in an extensive way to higher economic growth in any country. Thus, study of banking efficiency is very important for policy makers, industry leaders and many others who rely on banking sector.

A lot of research has been conducted over the past decade in the area of measuring efficiency of firms, companies, banks and other decision making units. Studies in the past used conventional ratios such as return on assets to evaluate efficiency. Most of these studies which look at the efficiency concentrate on cost, profit income or revenue efficiencies. Later research in the area used various measures of performance which include financial index (Wu et. al 2006), a non parametric approach- Data Envelopment Approach (DEA) (Wu, 2005), parametric approach and Stochastic Production Approach (SPA) (Radam et. al 2008). DEA is frequently used to measure relative efficiency of decision making units. DEA is defined by Charnes et al (1978) as a mathematical programming model applied to observations data that provide a new way of obtaining empirical estimate of relations such as the production functions or efficient production possibility surface which are considered to be the corner stone of modern economics. It is a non-parametric multiple input output efficiency technique that measures the relative efficiency of decision making units.

CCR-Model was introduced by Charnes, Cooper and Rhodes (1978). This model measures the efficiency of each DMU which is obtained as a maximum of the ratio of total sum of weighted outputs to total sum of weighted inputs. Consequently, the efficiency can be defined as follow.

\section{Efficiency $=\frac{\text { Weighted sum of outputs }}{\text { Weighted sum of inputs }}$}

The weights for the ratio are determined by the restriction that the similar ratios for every DMU have to be less than or equal to unity, thus reducing multiple inputs and outputs to a single "virtual" input and single "virtual" output without requiring pre-assigned weights. Therefore, the efficiency score is a function of the weights or the "virtual" input-output combination. Suppose that there are n DMUs, each with $\mathrm{n}$ inputs and $\mathrm{s}$ outputs, relative efficiency score of a given $\mathrm{DMU}_{0}$ is obtained by solving the following linear programming model.

$\max \left(\theta=\frac{\sum_{r=1}^{s} u_{r} y_{r 0}}{\sum_{i=1}^{m} v_{i} x_{i 0}}\right)$

subject to

$$
\frac{\sum_{r=1}^{s} u_{r} y_{r j}}{\sum_{i=1}^{m} v_{i} x_{i j}} \leq 1 ; j=1,2, \ldots, n
$$


where

$v_{i} \geq 0 ; i=1,2, \ldots, m$

$u_{i} \geq 0 ; r=1,2, \ldots, s$

and $x_{i j}=$ the amount of input $i$ utilized by the jth DMU

$y_{r j}=$ the amount of output $\mathrm{r}$ produced by the jth DMU

$v_{i}=$ weight given to input $\mathrm{i}$

$u_{r}=$ weight given to output $r$

Following the Charnes - Cooper transformation (1962), one can select a representative solution (v,u) for which

$$
\sum_{r=1}^{m} v_{i} x_{i 0}=1
$$

Hence, the denominator in the efficiency score $\theta$ shown above is set equal to one, the transformed linear programming model for $D M U_{0}$ can be written as follow.

$\operatorname{Max} \theta=\sum_{r=1}^{s} u_{r} y_{r 0}$

Subject to, $\sum_{r=1}^{s} u_{r} y_{r j}-\sum_{i=1}^{m} v_{i} x_{i j} \leq 0 ; j=1,2, \ldots, n$

And $\quad \sum_{i=1}^{m} v_{i} x_{i 0}=1$

$v_{i} \geq 0 ; i=1,2, \ldots, m$

$u_{i} \geq 0 ; r=1,2, \ldots, s$

The linear programming model shown above will be run $\mathrm{n}$ times in identifying the relative efficiency score of all the DMUs. Each DMU selects input and output weights that maximize its efficiency score. Generally, a DMU is considered to be efficient if it obtains a score of 1.00 , implying $100 \%$ efficiency; whereas a score of less than 1.00 implies that it is relatively inefficient.

\section{Literature Review}

Avkiran used DEA model, taking interest expense and non-interest expense as input variable and interest income and non-interest income as output variables to examine the efficiency of Australian trading banks for the period 1986 to 1995 and found that their efficiency rose in the post regulation period and acquiring banks were more efficient than target banks. Chen and Yeh (1998) calculated the operating efficiencies of 34 commercial banks of Taiwan's banks using the DEA model where in input variables included staff employed, interest expense and output variables include loans investment and interest revenue, non-interest revenue and bank assets. The author concluded that a bank with better efficiency does not always mean that it has better effectiveness.

In the case of Turkish Banks, (Mehmet Hasan Eken Suleyman Kale, A J B M vol. 5(3) PP 889-901, 4 Feb, 2011), it is apparent that branch size and scale efficiency are related to each other. As branch size increases scale efficiency increases too and after the most productive scale size, however, as the size increases efficiency decreases. Al-Shammari and Salimi (1998) have examined the comparative operating efficiency of Jordanian commercial banks from 1991-1994 using a modified version of DEA and found that the majority banks were fairly inefficient over the period 1991-1994. Noulas (2001) employed both DEA model and the traditional approach to study the effect of banking deregulation on private and public owned banks. The interest expense and non-interest expense were the input variable and interest revenue and non-interest revenue were the output variables. The result reveals that the state banks were less efficient than the private banks and the gap widened during the study period.

In India also, several studies have been carried out on Efficiency Analysis using DEA approach. Bhattacharya et al (1997) used DEA to measure the productive efficiency of Indian commercial banks in the late 80 s to early 90 s and studied the impact of policy on liberalizing measures taken in 1980s on the performance of various categories of banks. They found that Indian Public banks were the best performing banks as the banking sector was overwhelmingly dominated by Indian public sector banks while the new private sector banks were yet to emerge fully in the Indian banking scenario.

Sathye (2001) studied the relative efficiency of Indian banks in late 1990s and compared the efficiency of Indian banks with that of banks of other countries. He found that public sector banks have a higher mean efficiency score as compared to the private sector banks in India, but found mixed results when comparing public banks and foreign commercial banks in India. He also found that most banks on efficient frontier are foreign owned.

Rammohan and Ray (2004) compared the revenue maximizing efficiency of public, private and foreign banks in India using physical quantities of inputs and outputs in 1990s with deposits and operating costs as input and loans, investment and other income as outputs. They found that public sector banks were significantly better 
than private banks on revenue maximization efficiency, but between public and private sector banks the difference in efficiency was not significant.

Shanmugam and Das (2004) studied banking efficiency using a Stochastic Frontier Production function model during the reform model period 1992-99. The study considers the input variables (viz deposits, borrowings, labour and fixed assets) and four output variables (viz net interest income, non-interest income, credits and investments). They found that deposits are dominant in producing all outputs and technical efficiency of raising interest margin is varied across the banks. In particular they found that reform measures introduced since 1992 have not helped the banks in raising their interest margin. Also, in general, they found that private foreign banks performed better than public banks.

Sanjeev (2006) studied efficiency of private, public and foreign banks operating in India during the period 1997-2001 using DEA. He also studied if any relationship can be established between the efficiency and non-performing assets of the bank. He found that there is an increase in efficiency in post reform period and that non-performing assets and efficiency are negatively related.

Noulas and Ketkar (1996) measured the efficiency of public sector banks of India by using the Data Envelopment Analysis. The study considered 18 public sector banks and the necessary information for analysis have been collected from the RBI publications for the year 1993. The study identified that pure technical efficiency was 1.5 percent and scale inefficiency was 2.25 percent and none of the banks were operating under decreasing returns to scale.

\section{Research Methodology}

The present study considers ten commercial banks as decision making units. Out of these, five are public sector banks and other five are private sector banks. Selecting various inputs and outputs is a challenging task in the model because they can be misleading in nature. This paper considers three inputs which aredeposits, number of employees, operating expenses and the three outputs are investments, other income and advances. In this study the data related to various input and outputs over the period $2006-2010$ have been taken from RBI website (www.rbi.org.in). The ten decision making units (banks) considered in the present study are as follows:

\begin{tabular}{|c|c|}
\hline Public Sector Banks & Private Sector Banks \\
\hline State Bank of India(A) & HDFC Bank(F) \\
\hline Punjab National Bank(B) & Axis Bank(G) \\
\hline Canara Bank(C) $($ ICICI Bank(H) \\
\hline Vijaya Bank(D) & Yes Bank(I) \\
\hline Allahabad Bank(E) & Kotak Mahindra Bank(J) \\
\hline
\end{tabular}

The Linear Programming model formulated for the year $\mathbf{2 0 1 0}$ is as:

1. $\quad$ The L.P. for DMU A (SBI) is:

$<\mathrm{A}>\max \theta=\mathrm{u}$

Subject to $804116 \mathrm{v}_{1}+200299 \mathrm{v}_{2}+20319 \mathrm{v}_{3}=1$

$285790 \mathrm{u}_{1}+14968 \mathrm{u}_{2}+631914 \mathrm{u}_{3}<=804116 \mathrm{v}_{1}+200299 \mathrm{v}_{2}+20319 \mathrm{v}_{3}$

$77724 \mathrm{u}_{1}+3565 \mathrm{u}_{2}+186601 \mathrm{u}_{3}<=249330 \mathrm{v}_{1}+53417 \mathrm{v}_{2}+4762 \mathrm{v}_{3}$

$38429 \mathrm{u}_{1}+1516 \mathrm{u}_{2}+71605 \mathrm{u}_{3}<=106056 \mathrm{v}_{1}+20959 \mathrm{v}_{2}+1618 \mathrm{v}_{3}$

$69677 \mathrm{u}_{1}+2858 \mathrm{u}_{2}+169335 \mathrm{u}_{3}<=234651 \mathrm{v}_{1}+43380 \mathrm{v}_{2}+3478 \mathrm{v}_{3}$

$21107 \mathrm{u}_{1}+679 \mathrm{u}_{2}+41522 \mathrm{u}_{3}<=61932 \mathrm{v}_{1}+11565 \mathrm{v}_{2}+1072 \mathrm{v}_{3}$

$58608 \mathrm{u}_{1}+3808 \mathrm{u}_{2}+125831 \mathrm{u}_{3}<=167404 \mathrm{v}_{1}+51888 \mathrm{v}_{2}+5764 \mathrm{v}_{3}$

$55975 \mathrm{u}_{1}+3946 \mathrm{u}_{2}+104343 \mathrm{u}_{3}<=141300 \mathrm{v}_{1}+21640 \mathrm{v}_{2}+3710 \mathrm{v}_{3}$

$12513 \mathrm{u}_{1}+628 \mathrm{u}_{2}+20775 \mathrm{u}_{3}<=23886 \mathrm{v}_{1}+8632 \mathrm{v}_{2}+1189 \mathrm{v}_{3}$

$120893 \mathrm{u}_{1}+7478 \mathrm{u}_{2}+181206 \mathrm{u}_{3}<=202017 \mathrm{v}_{1}+35256 \mathrm{v}_{2}+5860 \mathrm{v}_{3}$

$10210 \mathrm{u}_{1}+576 \mathrm{u}_{2}+22193 \mathrm{u}_{3}<=26799 \mathrm{v}_{1}+3034 \mathrm{v}_{2}+500 \mathrm{v}_{3}$

Where all variables are constrained to be non-negative $\& \mathrm{u}_{1}, \mathrm{u}_{2}, \mathrm{u}_{3}$ are output variable weight $\& \mathrm{v}_{1}, \mathrm{v}_{2}, \mathrm{v}_{3}$ are input variable weight.

2. The L.P. for DMU B (PNB) is:

$<\mathrm{B}>\max \theta=\mathrm{u}$

Subject to $249330 \mathrm{v}_{1}+53417 \mathrm{v}_{2}+4762 \mathrm{v}_{3}=1$

$285790 \mathrm{u}_{1}+14968 \mathrm{u}_{2}+631914 \mathrm{u}_{3}<=804116 \mathrm{v}_{1}+200299 \mathrm{v}_{2}+20319 \mathrm{v}_{3}$

$77724 \mathrm{u}_{1}+3565 \mathrm{u}_{2}+186601 \mathrm{u}_{3}<=249330 \mathrm{v}_{1}+53417 \mathrm{v}_{2}+4762 \mathrm{v}_{3}$

$38429 \mathrm{u}_{1}+1516 \mathrm{u}_{2}+71605 \mathrm{u}_{3}<=106056 \mathrm{v}_{1}+20959 \mathrm{v}_{2}+1618 \mathrm{v}_{3}$

$69677 \mathrm{u}_{1}+2858 \mathrm{u}_{2}+169335 \mathrm{u}_{3}<=234651 \mathrm{v}_{1}+43380 \mathrm{v}_{2}+3478 \mathrm{v}_{3}$

$21107 \mathrm{u}_{1}+679 \mathrm{u}_{2}+41522 \mathrm{u}_{3}<=61932 \mathrm{v}_{1}+11565 \mathrm{v}_{2}+1072 \mathrm{v}_{3}$

$58608 \mathrm{u}_{1}+3808 \mathrm{u}_{2}+125831 \mathrm{u}_{3}<=167404 \mathrm{v}_{1}+51888 \mathrm{v}_{2}+5764 \mathrm{v}_{3}$

$55975 \mathrm{u}_{1}+3946 \mathrm{u}_{2}+104343 \mathrm{u}_{3}<=141300 \mathrm{v}_{1}+21640 \mathrm{v}_{2}+3710 \mathrm{v}_{3}$ 
$12513 \mathrm{u}_{1}+628 \mathrm{u}_{2}+20775 \mathrm{u}_{3}<=23886 \mathrm{v}_{1}+8632 \mathrm{v}_{2}+1189 \mathrm{v}_{3}$

$120893 \mathrm{u}_{1}+7478 \mathrm{u}_{2}+181206 \mathrm{u}_{3}<=202017 \mathrm{v}_{1}+35256 \mathrm{v}_{2}+5860 \mathrm{v}_{3}$

$10210 \mathrm{u}_{1}+576 \mathrm{u}_{2}+22193 \mathrm{u}_{3}<=26799 \mathrm{v}_{1}+3034 \mathrm{v}_{2}+500 \mathrm{v}_{3}$

Where all variables are constrained to be non negative $\& \mathrm{u}_{1}, \mathrm{u}_{2}, \mathrm{u}_{3}$ are output variable weight $\& \mathrm{v}_{1}, \mathrm{v}_{2}, \mathrm{v}_{3}$ are input variable weight.

\section{The L.P. for DMU C (Allahabad Bank) is:}

$<\mathrm{C}>\max \theta=\mathrm{u}$

Subject to $106056 \mathrm{v}_{1}+20959 \mathrm{v}_{2}+1618 \mathrm{v}_{3}=1$

$285790 u_{1}+14968 u_{2}+631914 u_{3}<=804116 v_{1}+200299 v_{2}+20319 v_{3}$

$77724 \mathrm{u}_{1}+3565 \mathrm{u}_{2}+186601 \mathrm{u}_{3}<=249330 \mathrm{v}_{1}+53417 \mathrm{v}_{2}+4762 \mathrm{v}_{3}$

$38429 \mathrm{u}_{1}+1516 \mathrm{u}_{2}+71605 \mathrm{u}_{3}<=106056 \mathrm{v}_{1}+20959 \mathrm{v}_{2}+1618 \mathrm{v}_{3}$

$69677 \mathrm{u}_{1}+2858 \mathrm{u}_{2}+169335 \mathrm{u}_{3}<=234651 \mathrm{v}_{1}+43380 \mathrm{v}_{2}+3478 \mathrm{v}_{3}$

$21107 \mathrm{u}_{1}+679 \mathrm{u}_{2}+41522 \mathrm{u}_{3}<=61932 \mathrm{v}_{1}+11565 \mathrm{v}_{2}+1072 \mathrm{v}_{3}$

$58608 \mathrm{u}_{1}+3808 \mathrm{u}_{2}+125831 \mathrm{u}_{3}<=167404 \mathrm{v}_{1}+51888 \mathrm{v}_{2}+5764 \mathrm{v}_{3}$

$55975 \mathrm{u}_{1}+3946 \mathrm{u}_{2}+104343 \mathrm{u}_{3}<=141300 \mathrm{v}_{1}+21640 \mathrm{v}_{2}+3710 \mathrm{v}_{3}$

$12513 \mathrm{u}_{1}+628 \mathrm{u}_{2}+20775 \mathrm{u}_{3}<=23886 \mathrm{v}_{1}+8632 \mathrm{v}_{2}+1189 \mathrm{v}_{3}$

$120893 \mathrm{u}_{1}+7478 \mathrm{u}_{2}+181206 \mathrm{u}_{3}<=202017 \mathrm{v}_{1}+35256 \mathrm{v}_{2}+5860 \mathrm{v}_{3}$

$10210 \mathrm{u}_{1}+576 \mathrm{u}_{2}+22193 \mathrm{u}_{3}<=26799 \mathrm{v}_{1}+3034 \mathrm{v}_{2}+500 \mathrm{v}_{3}$

Where all variables are constrained to be non negative $\& \mathrm{u}_{1}, \mathrm{u}_{2}, \mathrm{u}_{3}$ are output variable weight $\& \mathrm{v}_{1}, \mathrm{v}_{2}, \mathrm{v}_{3}$ are input variable weight.

4. The L.P. for DMU D (Canara Bank) is:

$<\mathrm{D}>\max \theta=\mathrm{u}$

Subject to $234651 v_{1}+43380 v_{2}+3478 v_{3}=1$

$285790 u_{1}+14968 u_{2}+631914 u_{3}<=804116 v_{1}+200299 v_{2}+20319 v_{3}$

$77724 \mathrm{u}_{1}+3565 \mathrm{u}_{2}+186601 \mathrm{u}_{3}<=249330 \mathrm{v}_{1}+53417 \mathrm{v}_{2}+4762 \mathrm{v}_{3}$

$38429 \mathrm{u}_{1}+1516 \mathrm{u}_{2}+71605 \mathrm{u}_{3}<=106056 \mathrm{v}_{1}+20959 \mathrm{v}_{2}+1618 \mathrm{v}_{3}$

$69677 \mathrm{u}_{1}+2858 \mathrm{u}_{2}+169335 \mathrm{u}_{3}<=234651 \mathrm{v}_{1}+43380 \mathrm{v}_{2}+3478 \mathrm{v}_{3}$

$21107 \mathrm{u}_{1}+679 \mathrm{u}_{2}+41522 \mathrm{u}_{3}<=61932 \mathrm{v}_{1}+11565 \mathrm{v}_{2}+1072 \mathrm{v}_{3}$

$58608 \mathrm{u}_{1}+3808 \mathrm{u}_{2}+125831 \mathrm{u}_{3}<=167404 \mathrm{v}_{1}+51888 \mathrm{v}_{2}+5764 \mathrm{v}_{3}$

$55975 \mathrm{u}_{1}+3946 \mathrm{u}_{2}+104343 \mathrm{u}_{3}<=141300 \mathrm{v}_{1}+21640 \mathrm{v}_{2}+3710 \mathrm{v}_{3}$

$12513 \mathrm{u}_{1}+628 \mathrm{u}_{2}+20775 \mathrm{u}_{3}<=23886 \mathrm{v}_{1}+8632 \mathrm{v}_{2}+1189 \mathrm{v}_{3}$

$120893 \mathrm{u}_{1}+7478 \mathrm{u}_{2}+181206 \mathrm{u}_{3}<=202017 \mathrm{v}_{1}+35256 \mathrm{v}_{2}+5860 \mathrm{v}_{3}$

$10210 \mathrm{u}_{1}+576 \mathrm{u}_{2}+22193 \mathrm{u}_{3}<=26799 \mathrm{v}_{1}+3034 \mathrm{v}_{2}+500 \mathrm{v}_{3}$

Where all variables are constrained to be non negative $\& \mathrm{u}_{1}, \mathrm{u}_{2}, \mathrm{u}_{3}$ are output variable weight $\& \mathrm{v}_{1}, \mathrm{v}_{2}, \mathrm{v}_{3}$ are input variable weight.

5. The L.P. for DMU E (Vijaya Bank) is:

$<\mathrm{E}>\max \theta=\mathrm{u}$

Subject to $61932 \mathrm{v}_{1}+11565 \mathrm{v}_{2}+1072 \mathrm{v}_{3}=1$

$285790 \mathrm{u}_{1}+14968 \mathrm{u}_{2}+631914 \mathrm{u}_{3}<=804116 \mathrm{v}_{1}+200299 \mathrm{v}_{2}+20319 \mathrm{v}_{3}$

$77724 \mathrm{u}_{1}+3565 \mathrm{u}_{2}+186601 \mathrm{u}_{3}<=249330 \mathrm{v}_{1}+53417 \mathrm{v}_{2}+4762 \mathrm{v}_{3}$

$38429 \mathrm{u}_{1}+1516 \mathrm{u}_{2}+71605 \mathrm{u}_{3}<=106056 \mathrm{v}_{1}+20959 \mathrm{v}_{2}+1618 \mathrm{v}_{3}$

$69677 \mathrm{u}_{1}+2858 \mathrm{u}_{2}+169335 \mathrm{u}_{3}<=234651 \mathrm{v}_{1}+43380 \mathrm{v}_{2}+3478 \mathrm{v}_{3}$

$21107 \mathrm{u}_{1}+679 \mathrm{u}_{2}+41522 \mathrm{u}_{3}<=61932 \mathrm{v}_{1}+11565 \mathrm{v}_{2}+1072 \mathrm{v}_{3}$

$58608 \mathrm{u}_{1}+3808 \mathrm{u}_{2}+125831 \mathrm{u}_{3}<=167404 \mathrm{v}_{1}+51888 \mathrm{v}_{2}+5764 \mathrm{v}_{3}$

$55975 \mathrm{u}_{1}+3946 \mathrm{u}_{2}+104343 \mathrm{u}_{3}<=141300 \mathrm{v}_{1}+21640 \mathrm{v}_{2}+3710 \mathrm{v}_{3}$

$12513 \mathrm{u}_{1}+628 \mathrm{u}_{2}+20775 \mathrm{u}_{3}<=23886 \mathrm{v}_{1}+8632 \mathrm{v}_{2}+1189 \mathrm{v}_{3}$

$120893 \mathrm{u}_{1}+7478 \mathrm{u}_{2}+181206 \mathrm{u}_{3}<=202017 \mathrm{v}_{1}+35256 \mathrm{v}_{2}+5860 \mathrm{v}_{3}$

$10210 \mathrm{u}_{1}+576 \mathrm{u}_{2}+22193 \mathrm{u}_{3}<=26799 \mathrm{v}_{1}+3034 \mathrm{v}_{2}+500 \mathrm{v}_{3}$

Where all variables are constrained to be non negative $\& \mathrm{u}_{1}, \mathrm{u}_{2}, \mathrm{u}_{3}$ are output variable weight $\& \mathrm{v}_{1}, \mathrm{v}_{2}, \mathrm{v}_{3}$ are input variable weight.

6. The L.P. for DMU F (HDFC) is:

$<\mathrm{F}>\max \theta=\mathrm{u}$

Subject to $167404 \mathrm{v}_{1}+51888 \mathrm{v}_{2}+5764 \mathrm{v}_{3}=1$

$285790 \mathrm{u}_{1}+14968 \mathrm{u}_{2}+631914 \mathrm{u}_{3}<=804116 \mathrm{v}_{1}+200299 \mathrm{v}_{2}+20319 \mathrm{v}_{3}$

$77724 \mathrm{u}_{1}+3565 \mathrm{u}_{2}+186601 \mathrm{u}_{3}<=249330 \mathrm{v}_{1}+53417 \mathrm{v}_{2}+4762 \mathrm{v}_{3}$

$38429 \mathrm{u}_{1}+1516 \mathrm{u}_{2}+71605 \mathrm{u}_{3}<=106056 \mathrm{v}_{1}+20959 \mathrm{v}_{2}+1618 \mathrm{v}_{3}$

$69677 \mathrm{u}_{1}+2858 \mathrm{u}_{2}+169335 \mathrm{u}_{3}<=234651 \mathrm{v}_{1}+43380 \mathrm{v}_{2}+3478 \mathrm{v}_{3}$ 
$21107 \mathrm{u}_{1}+679 \mathrm{u}_{2}+41522 \mathrm{u}_{3}<=61932 \mathrm{v}_{1}+11565 \mathrm{v}_{2}+1072 \mathrm{v}_{3}$

$58608 \mathrm{u}_{1}+3808 \mathrm{u}_{2}+125831 \mathrm{u}_{3}<=167404 \mathrm{v}_{1}+51888 \mathrm{v}_{2}+5764 \mathrm{v}_{3}$

$55975 \mathrm{u}_{1}+3946 \mathrm{u}_{2}+104343 \mathrm{u}_{3}<=141300 \mathrm{v}_{1}+21640 \mathrm{v}_{2}+3710 \mathrm{v}_{3}$

$12513 \mathrm{u}_{1}+628 \mathrm{u}_{2}+20775 \mathrm{u}_{3}<=23886 \mathrm{v}_{1}+8632 \mathrm{v}_{2}+1189 \mathrm{v}_{3}$

$120893 \mathrm{u}_{1}+7478 \mathrm{u}_{2}+181206 \mathrm{u}_{3}<=202017 \mathrm{v}_{1}+35256 \mathrm{v}_{2}+5860 \mathrm{v}_{3}$

$10210 u_{1}+576 u_{2}+22193 u_{3}<=26799 v_{1}+3034 v_{2}+500 v_{3}$

Where all variables are constrained to be non negative $\& \mathrm{u}_{1}, \mathrm{u}_{2}, \mathrm{u}_{3}$ are output variable weight $\& \mathrm{v}_{1}, \mathrm{v}_{2}, \mathrm{v}_{3}$ are input variable weight.

7. The L.P. for DMU G (Axis Bank) is:

$<\mathrm{G}>\max \theta=\mathrm{u}$

Subject to $141300 \mathrm{v}_{1}+21640 \mathrm{v}_{2}+3710 \mathrm{v}_{3}=1$

$285790 u_{1}+14968 u_{2}+631914 u_{3}<=804116 v_{1}+200299 v_{2}+20319 v_{3}$

$77724 \mathrm{u}_{1}+3565 \mathrm{u}_{2}+186601 \mathrm{u}_{3}<=249330 \mathrm{v}_{1}+53417 \mathrm{v}_{2}+4762 \mathrm{v}_{3}$

$38429 \mathrm{u}_{1}+1516 \mathrm{u}_{2}+71605 \mathrm{u}_{3}<=106056 \mathrm{v}_{1}+20959 \mathrm{v}_{2}+1618 \mathrm{v}_{3}$

$69677 \mathrm{u}_{1}+2858 \mathrm{u}_{2}+169335 \mathrm{u}_{3}<=234651 \mathrm{v}_{1}+43380 \mathrm{v}_{2}+3478 \mathrm{v}_{3}$

$21107 \mathrm{u}_{1}+679 \mathrm{u}_{2}+41522 \mathrm{u}_{3}<=61932 \mathrm{v}_{1}+11565 \mathrm{v}_{2}+1072 \mathrm{v}_{3}$

$58608 \mathrm{u}_{1}+3808 \mathrm{u}_{2}+125831 \mathrm{u}_{3}<=167404 \mathrm{v}_{1}+51888 \mathrm{v}_{2}+5764 \mathrm{v}_{3}$

$55975 \mathrm{u}_{1}+3946 \mathrm{u}_{2}+104343 \mathrm{u}_{3}<=141300 \mathrm{v}_{1}+21640 \mathrm{v}_{2}+3710 \mathrm{v}_{3}$

$12513 \mathrm{u}_{1}+628 \mathrm{u}_{2}+20775 \mathrm{u}_{3}<=23886 \mathrm{v}_{1}+8632 \mathrm{v}_{2}+1189 \mathrm{v}_{3}$

$120893 \mathrm{u}_{1}+7478 \mathrm{u}_{2}+181206 \mathrm{u}_{3}<=202017 \mathrm{v}_{1}+35256 \mathrm{v}_{2}+5860 \mathrm{v}_{3}$

$10210 \mathrm{u}_{1}+576 \mathrm{u}_{2}+22193 \mathrm{u}_{3}<=26799 \mathrm{v}_{1}+3034 \mathrm{v}_{2}+500 \mathrm{v}_{3}$

Where all variables are constrained to be non negative $\& \mathrm{u}_{1}, \mathrm{u}_{2}, \mathrm{u}_{3}$ are output variable weight $\& \mathrm{v}_{1}, \mathrm{v}_{2}, \mathrm{v}_{3}$ are input variable weight.

8. The L.P. for DMU H (Kotak Mahindra Bank) is:

$<\mathrm{H}>\max \theta=\mathrm{u}$

Subject to $23886 \mathrm{v}_{1}+8632 \mathrm{v}_{2}+1189 \mathrm{v}_{3}=1$

$285790 \mathrm{u}_{1}+14968 \mathrm{u}_{2}+631914 \mathrm{u}_{3}<=804116 \mathrm{v}_{1}+200299 \mathrm{v}_{2}+20319 \mathrm{v}_{3}$

$77724 \mathrm{u}_{1}+3565 \mathrm{u}_{2}+186601 \mathrm{u}_{3}<=249330 \mathrm{v}_{1}+53417 \mathrm{v}_{2}+4762 \mathrm{v}_{3}$

$38429 \mathrm{u}_{1}+1516 \mathrm{u}_{2}+71605 \mathrm{u}_{3}<=106056 \mathrm{v}_{1}+20959 \mathrm{v}_{2}+1618 \mathrm{v}_{3}$

$69677 \mathrm{u}_{1}+2858 \mathrm{u}_{2}+169335 \mathrm{u}_{3}<=234651 \mathrm{v}_{1}+43380 \mathrm{v}_{2}+3478 \mathrm{v}_{3}$

$21107 \mathrm{u}_{1}+679 \mathrm{u}_{2}+41522 \mathrm{u}_{3}<=61932 \mathrm{v}_{1}+11565 \mathrm{v}_{2}+1072 \mathrm{v}_{3}$

$58608 \mathrm{u}_{1}+3808 \mathrm{u}_{2}+125831 \mathrm{u}_{3}<=167404 \mathrm{v}_{1}+51888 \mathrm{v}_{2}+5764 \mathrm{v}_{3}$

$55975 \mathrm{u}_{1}+3946 \mathrm{u}_{2}+104343 \mathrm{u}_{3}<=141300 \mathrm{v}_{1}+21640 \mathrm{v}_{2}+3710 \mathrm{v}_{3}$

$12513 \mathrm{u}_{1}+628 \mathrm{u}_{2}+20775 \mathrm{u}_{3}<=23886 \mathrm{v}_{1}+8632 \mathrm{v}_{2}+1189 \mathrm{v}_{3}$

$120893 \mathrm{u}_{1}+7478 \mathrm{u}_{2}+181206 \mathrm{u}_{3}<=202017 \mathrm{v}_{1}+35256 \mathrm{v}_{2}+5860 \mathrm{v}_{3}$

$10210 \mathrm{u}_{1}+576 \mathrm{u}_{2}+22193 \mathrm{u}_{3}<=26799 \mathrm{v}_{1}+3034 \mathrm{v}_{2}+500 \mathrm{v}_{3}$

Where all variables are constrained to be non negative $\& \mathrm{u}_{1}, \mathrm{u}_{2}, \mathrm{u}_{3}$ are output variable weight $\& \mathrm{v}_{1}, \mathrm{v}_{2}, \mathrm{v}_{3}$ are input variable weight.

9. The L.P. for DMU I (ICICI Bank) is:

$<\mathrm{I}>\max \theta=\mathrm{u}$

Subject to $202017 \mathrm{v}_{1}+35256 \mathrm{v}_{2}+5860 \mathrm{v}_{3}=1$

$285790 \mathrm{u}_{1}+14968 \mathrm{u}_{2}+631914 \mathrm{u}_{3}<=804116 \mathrm{v}_{1}+200299 \mathrm{v}_{2}+20319 \mathrm{v}_{3}$

$77724 \mathrm{u}_{1}+3565 \mathrm{u}_{2}+186601 \mathrm{u}_{3}<=249330 \mathrm{v}_{1}+53417 \mathrm{v}_{2}+4762 \mathrm{v}_{3}$

$38429 \mathrm{u}_{1}+1516 \mathrm{u}_{2}+71605 \mathrm{u}_{3}<=106056 \mathrm{v}_{1}+20959 \mathrm{v}_{2}+1618 \mathrm{v}_{3}$

$69677 \mathrm{u}_{1}+2858 \mathrm{u}_{2}+169335 \mathrm{u}_{3}<=234651 \mathrm{v}_{1}+43380 \mathrm{v}_{2}+3478 \mathrm{v}_{3}$

$21107 \mathrm{u}_{1}+679 \mathrm{u}_{2}+41522 \mathrm{u}_{3}<=61932 \mathrm{v}_{1}+11565 \mathrm{v}_{2}+1072 \mathrm{v}_{3}$

$58608 \mathrm{u}_{1}+3808 \mathrm{u}_{2}+125831 \mathrm{u}_{3}<=167404 \mathrm{v}_{1}+51888 \mathrm{v}_{2}+5764 \mathrm{v}_{3}$

$55975 \mathrm{u}_{1}+3946 \mathrm{u}_{2}+104343 \mathrm{u}_{3}<=141300 \mathrm{v}_{1}+21640 \mathrm{v}_{2}+3710 \mathrm{v}_{3}$

$12513 \mathrm{u}_{1}+628 \mathrm{u}_{2}+20775 \mathrm{u}_{3}<=23886 \mathrm{v}_{1}+8632 \mathrm{v}_{2}+1189 \mathrm{v}_{3}$

$120893 \mathrm{u}_{1}+7478 \mathrm{u}_{2}+181206 \mathrm{u}_{3}<=202017 \mathrm{v}_{1}+35256 \mathrm{v}_{2}+5860 \mathrm{v}_{3}$

$10210 \mathrm{u}_{1}+576 \mathrm{u}_{2}+22193 \mathrm{u}_{3}<=26799 \mathrm{v}_{1}+3034 \mathrm{v}_{2}+500 \mathrm{v}_{3}$ 
Where all variables are constrained to be non negative $\& \mathrm{u}_{1}, \mathrm{u}_{2}, \mathrm{u}_{3}$ are output variable weight $\& \mathrm{v}_{1}, \mathrm{v}_{2}, \mathrm{v}_{3}$ are input variable weight.

10. The L.P. for DMU J (Yes Bank) is:

$<\mathrm{J}>\max \theta=\mathrm{u}$

Subject to $26799 \mathrm{v}_{1}+3034 \mathrm{v}_{2}+500 \mathrm{v}_{3}=1$

$$
285790 u_{1}+14968 u_{2}+631914 u_{3}<=804116 v_{1}+200299 v_{2}+20319 v_{3}
$$

$77724 \mathrm{u}_{1}+3565 \mathrm{u}_{2}+186601 \mathrm{u}_{3}<=249330 \mathrm{v}_{1}+53417 \mathrm{v}_{2}+4762 \mathrm{v}_{3}$

$38429 \mathrm{u}_{1}+1516 \mathrm{u}_{2}+71605 \mathrm{u}_{3}<=106056 \mathrm{v}_{1}+20959 \mathrm{v}_{2}+1618 \mathrm{v}_{3}$

$69677 \mathrm{u}_{1}+2858 \mathrm{u}_{2}+169335 \mathrm{u}_{3}<=234651 \mathrm{v}_{1}+43380 \mathrm{v}_{2}+3478 \mathrm{v}_{3}$

$21107 \mathrm{u}_{1}+679 \mathrm{u}_{2}+41522 \mathrm{u}_{3}<=61932 \mathrm{v}_{1}+11565 \mathrm{v}_{2}+1072 \mathrm{v}_{3}$

$58608 \mathrm{u}_{1}+3808 \mathrm{u}_{2}+125831 \mathrm{u}_{3}<=167404 \mathrm{v}_{1}+51888 \mathrm{v}_{2}+5764 \mathrm{v}_{3}$

$55975 \mathrm{u}_{1}+3946 \mathrm{u}_{2}+104343 \mathrm{u}_{3}<=141300 \mathrm{v}_{1}+21640 \mathrm{v}_{2}+3710 \mathrm{v}_{3}$

$12513 \mathrm{u}_{1}+628 \mathrm{u}_{2}+20775 \mathrm{u}_{3}<=23886 \mathrm{v}_{1}+8632 \mathrm{v}_{2}+1189 \mathrm{v}_{3}$

$120893 \mathrm{u}_{1}+7478 \mathrm{u}_{2}+181206 \mathrm{u}_{3}<=202017 \mathrm{v}_{1}+35256 \mathrm{v}_{2}+5860 \mathrm{v}_{3}$

$10210 u_{1}+576 u_{2}+22193 u_{3}<=26799 v_{1}+3034 v_{2}+500 v_{3}$

Where all variables are considered to be non negative \& $\mathrm{u}_{1}, \mathrm{u}_{2}, \mathrm{u}_{3}$ are output variable weight $\& \mathrm{v}_{1}, \mathrm{v}_{2}, \mathrm{v}_{3}$ are input variable weight.

Similarly Linear Programming model is formulated for the year 2009, 2008, 2007 and2006.

The efficiency of various DMUs obtained using the CCR model is as follows:

Table 1: Technical Efficiency of Banks

\begin{tabular}{|l|l|l|l|l|l|l|}
\hline $\begin{array}{l}\text { Serial } \\
\text { Number }\end{array}$ & DMU (Banks) & $\begin{array}{l}\text { Efficiency } \\
\mathbf{2 0 0 6}\end{array}$ & $\begin{array}{l}\text { Efficiency } \\
\mathbf{2 0 0 7}\end{array}$ & $\begin{array}{l}\text { Efficiency } \\
\mathbf{2 0 0 8}\end{array}$ & $\begin{array}{l}\text { Efficiency } \\
\mathbf{2 0 0 9}\end{array}$ & $\begin{array}{l}\text { Efficiency } \\
\mathbf{2 0 1 0}\end{array}$ \\
\hline A & $\begin{array}{l}\text { State Bank of } \\
\text { India }\end{array}$ & 0.891611 & 0.9390914 & 0.980034 & 0.9582734 & 0.9010883 \\
\hline B & $\begin{array}{l}\text { Punjab National } \\
\text { Bank }\end{array}$ & 0.821143 & 0.8883662 & 0.943514 & 0.9225176 & 0.9005408 \\
\hline C & Allahabad Bank & 0.909731 & 1 & 1 & 1 & 1 \\
\hline D & Canara Bank & 1 & 1 & 0.966503 & 1 & 1 \\
\hline E & Vijaya Bank & 1 & 0.9750875 & 1 & 0.9475686 & 0.8946582 \\
\hline F & HDFC Bank & 0.985309 & 0.8937566 & 1 & 0.8436847 & 0.8379867 \\
\hline G & Axis Bank & 1 & 1 & 1 & 0.9714755 & 0.875286 \\
\hline H & $\begin{array}{l}\text { Kotak } \\
\text { Mahindra Bank }\end{array}$ & 1 & 1 & 1 & 1 & 0.9696454 \\
\hline I & ICICI Bank & 1 & & 1 & $\mathbf{1}$ & 1 \\
\hline J & Yes Bank & $\mathbf{1}$ & $\mathbf{1}$ & $\mathbf{1}$ & 1 \\
\hline
\end{tabular}

Table 2 Average Efficiency scores of Banks

\begin{tabular}{|l|l|l|}
\hline Serial No & Period & Average Efficiency score \\
\hline 1. & 2006 & 0.96078 \\
\hline 2. & 2007 & 0.96963 \\
\hline 3. & 2008 & 0.98900 \\
\hline 4. & 2009 & 0.96435 \\
\hline 5. & 2010 & 0.9379 \\
\hline
\end{tabular}

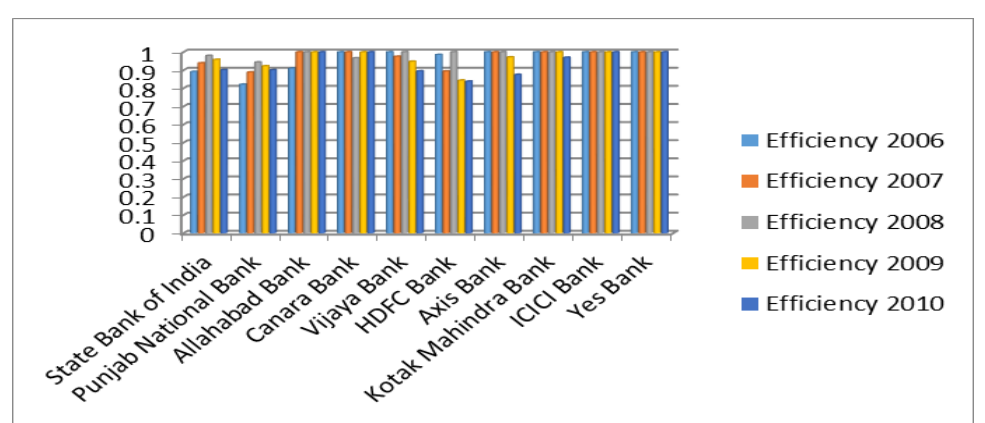

Figure 1: Efficiency trend Graph 2006 - 2010

\section{Conclusion}

- This study attempts to investigate the technical efficiency of Indian commercial banks during the period of 2006-2010. Tests for each year have been run, for ten Indian commercial banks. 
- The results suggest that the mean technical efficiency improved from 2006 to 2008 and then technical efficiency declined during the period 2008 to 2010. The result also shows that the banks like Allahabad bank, Canara bank, Kotak Mahindra bank, ICICI bank and Yes bank are very efficient and they have consistency in their performance.

- Banks like SBI, PNB and HDFC can be a matter of concern as their efficiency scores are below satisfactory level. The major factor resulting in the poor performance by these two banks is their huge amounts of deposits and operating expenses. Also, the excess number of employees is increasing their problems. Either these banks possess blocked/non-performing assets or are not able to make a set off between the deposits and advances. Being major banks in the country, they deserve to be paid a better attention of the regulators and the administrators.

- The performance of private sector banks is better than public sector banks during the period of investigation, 2006-2010.

\section{References:}

[1]. Ahn, T., Charnes, A., Cooper, W.W.(1989), An Note on the Efficiency Characterizations Obtained in Different DEA Models. Socio-Economic Planning Sciences, vol. 22, pp.253-257.

[2]. Avikaran, N.K., (2006), productivity Analysis in the service sector with Data Envelopment Analysis. Third Edition Australia.

[3]. Banker, R.D. and Natarajan, R.(2008). Evaluating Contextual Variables Affecting Productivity Using Data Envelopment Analysis. Operations Research, vol. 56, No.1, pp.48-58.

[4]. Banker, R.D. and Thrall, R.M.(1992).Estimating Most Productive Scale Size Using Data Envelopment Analysis. European Journal of Operational Research, vol. 62, pp.74-84.

[5]. Banker, R.D., (1984). Estimating most productive scale size using Data Envelopment Analysis. .European Journal of operational Research, vol.17, pp. 35-44.

[6]. Banker, R.D., Bardhan, I., Cooper, W.W.(1996). A Note On Return to Scale in DEA. European Journal of Operational Research, vol. 88 , pp.583-585.

[7]. Banker, R.D., Chang, H., Cooper, w.w.(1996).Equivalence and implementation of alternative methods of determining returns to scale in Data Envelopment Analysis. European Journal of Operational Research,vol. 89,pp.473-481.

[8]. ChamiLatha, Patil N, Mohanthy R.P. (2009). Measuring Efficiency in Indian cement industry using DEA. Industrial Engineering Journal, Vol.2, issue No-04 oct-2009, India.

[9]. Charnes, A., Cooper, W.W. (1991). DEA Usages and Interpretations reproduced in Proceedings of International Federation of Operational Research Societies 12th Triennial Conference, Athens, Greece, 1990.

[10]. Charnes, A., Cooper,W.W., Golany,B. and Seiford, L. (1985) Foundations of Data Envelopment Analysis for Pareto-Koopmans Efficient Empirical Production Functions.J Econom 30: 91-107.

[11]. Cooper, W.W., Seiford, L.M.and Tone, K. (2000). Data Envelopment Analysis: A comprehensive Text with Models, Applications, References and DEA-solver software Kluwer Academic Publishers: Boston.

[12]. Kuosmanen, T. (1999) some remarks on scale efficiency and returns to scale in DEA. Itelsinks school of Economics and Business Administration, Helsinki.

[13]. Millam, J.A. and Aldaz, N. (2004) Efficiency and technical change in inter-temporal inter-sectoral DEA. Journal of Productivity Analysis, vol.21, pp.7-23.

[14]. Nand Kumar and Archana Singh,(2014) Efficiency Analysis of Banks using DEA: A Review, International Journal of Advance Research and Innovation, Vol 1,pp.120-126.

[15]. Thore, S. Kozmetsky, G. and Phillips, F.(1994). DEA of financial statements Data: The US computer industry. The Journal of Productivity Analysis, vol. 5, pp. 229-248.

[16]. Tone, K.(1996). A Simple Characterization of Returns to Scale in DEA, Journal of the Operations Research Society of Japan, vol.39, pp.604-613.9 (Dec 2005), No.4, pp. 261-285.

[17]. Tone, K.(1997) A Slacks-based Measure of Efficiency in Data Envelopment Analysis, Research Reports, Graduate School of Policy Science, Saitama University, Urawa, Saitama, Japan, also forthcoming in European Journal of Operational Research.

[18]. Tone, Kaoru, Sahoo, B., Evaluating Cost Efficiency and Return to Scale in the Life Insurance Corporation of India using Data Envelopment Analysis. Socioeconomic Planning Sciences, Vol. 3

[19]. Tubene, S.L. (1997) Measuring Productivity across Nations using Data Envelopment Analysis. Ph.D. dissertation. USA: Dept. of Agricultural Economics, Kansas State University. Pp.1-154.

[20]. Wu, D. (D), Yang, Z., Liang, L.(2006 b).Using DEA.Nema Network Approach to evaluate Branch efficiency of a Large Canadian Bank. Expert syst App, vol. 31, No.1, pp. 108-115. 